Pneumologe 2011 - 8:215-216

DOI 10.1007/s10405-010-0437-7

Online publiziert: 7. Mai 2011

(c) Springer-Verlag 2011

\author{
R.C. Bittner \\ Institut für Diagnostische und Interventionelle Radiologie, \\ Helios Klinikum Emil von Behring, Berlin
}

\title{
Bildgebung in der Pneumologie
}

Die bildgebende Diagnostik mit radiologischen, nuklearmedizinischen und sonographischen Verfahren sowie die radiologischen Interventionen haben sich besonders in den letzten Jahren erheblich weiterentwickelt. Dabei war die Geschwindigkeit des technischen Fortschritts zeitweilig so hoch, dass es für nicht direkt Involvierte schwierig wurde, den Überblick zu behalten. Unter den mit wesentlichen Konsequenzen für die thorakale Diagnostik einhergehenden „corner stones“ der Entwicklung seien hier v. a. erwähnt: Mehrzeilen-Spiral-CT, Detektorradiographie, PET-CT sowie bildgesteuerte minimal-invasive Interventionen. Auch wir Radiologen und Nuklearmediziner selbst waren im Laufe unserer klinischen und wissenschaftlichen Tätigkeit von einigen dieser Fortschritte überrascht. In der kritischen Bewertung und Testung dieser Neuerungen wurde deutlich, dass es nicht leicht ist, das technisch Mögliche vom Sinnvollen bzw. Nutzbringenden zu unterscheiden und gleichzeitig ein Ausufern der Kosten durch diese neuen Techniken zu verhindern.

\section{(? Die bildgebende Diagnostik erfordert stets, das technisch Mögliche vom medizinisch Sinnvollen zu unterscheiden}

Daher macht es Sinn, den aktuellen Stand des Wissens und der Technik in der thorakalen Diagnostik und Intervention im Kontext zu sinnvollem Einsatz bzw. Abfolge derselben durch versierte Radiologen und Nuklearmediziner in kompakter Übersicht darzustellen. Es ist gelungen, für dieses Heft namhafte Experten für die einzelnen Beiträge zu gewinnen, die den
Lesern neben zahlreichen Detailinformationen übersichtliche Zusammenfassungen und damit den aktuellen Stand des radiologischen Expertenwissens in der thorakalen Diagnostik und minimal-invasiven radiologischen Intervention präsentieren.

Die bildgebende Diagnostik in der Klinik bewegt sich zwischen den Extremen maximaler Aufwand mit zum Teil erheblichen Kosten und möglichst weitgehender Vermeidung von Kosten durch Verzicht auf radiologische Verfahren. Da aus betriebswirtschaftlicher Sicht die Radiologie als Dienstleister ähnlich wie Labor oder Pathologie als Kostenverursacher angesehen wird, macht es absolut Sinn, die Radiologie in klinische Entscheidungsabläufe als ständigen Partner einzubinden. Hierdurch werden insbesondere durch zielgerichtete Diagnostik und die Vermeidung von redundanten Verfahren Zeit und Kosten eingespart.

So beschreibt der Beitrag von S. Diederich, Düsseldorf, die verschiedenen Möglichkeiten, mithilfe minimal-invasiver radiologischer Verfahren schneller zu erfolgreichen diagnostischen und therapeutischen Resultaten zu kommen. Hier können erhebliche Kosten u. a. auch durch Verkürzung der Liegezeit des Patienten eingespart werden. Die sofortige CT-gesteuerte Biopsie perkutan transthorakal bei nicht primär operablen, peripheren pulmonalen tumorsuspekten Herden ohne Wartezeit auf das Resultat der Bronchoskopie bei diesen Patienten wird in unserer Klinik schon länger erfolgreich praktiziert. Dieses Vorgehen wird zuvor einvernehmlich interdisziplinär in einer Tumorkonferenz vereinbart und terminiert. 
Den größten Einfluss auf die Therapie bei thorakalen Malignomen, insbesondere Lungenkarzinomen, hat derzeit die PET-CT. Das Verfahren und die resultierenden Konsequenzen werden von G.F. Förster et al., Berlin, ausführlich dargestellt. In der täglichen Routine ergeben sich nach über 3 Jahren angewandter PET-CT in der Lungenkarzinomdiagnostik in unserer Klinik erhebliche Therapieänderungen bei ca. einem Drittel der Patienten im Vergleich zur ursprünglichen klinischen Planung. Inzwischen werden in unserem Haus alle potenziell operablen Patienten mit Lungenkarzinom einer PET-CT unterzogen. Die diesbezügliche Beschlussfassung erfolgt in der mindestens 2-mal wöchentlich stattfindenden Tumorkonferenz, an der alle involvierten Disziplinen einschließlich Pathologie, Radiologie und Strahlentherapie teilnehmen.

Der Beitrag der hochauflösenden CT, kurz HRCT, für die thorakale Diagnostik wird weiterhin unterschätzt, v. a. in der Differenzialdiagnostik unklarer Lungenparenchymerkrankungen. Der Beitrag von D. Wormanns, Berlin, gibt hier exakte Hilfestellung in der Indikationsstellung zur HRCT.

Der Stellenwert der MRT in der thorakalen Diagnostik wird ebenfalls erheblich unterschätzt, legt man die Möglichkeiten des Verfahrens, die von J. Biederer et al., Kiel, hier ausführlich dargestellt werden, zugrunde. Nach meiner Einschätzung wird es dennoch schwierig bleiben, bis auf selektive Fälle einen routinemäßigen Einsatz des Verfahrens in der thorakalen, insbesondere der pulmonalen Diagnostik zu etablieren, da die Verfügbarkeit der Geräte angesichts der übrigen stetig steigenden Indikationen für eine MRT nicht wirklich gewährleistet sein wird.

Die neben der dominierenden PETCT in der Thoraxdiagnostik noch wichtigen nuklearmedizinischen Techniken werden von S. Dresel et al., Berlin, aktualisiert und ausführlich erläutert.

Eine zusammenfassende Bewertung und aktuelle Standortbestimmung für die gängigen bildgebenden Routineverfahren in der Thoraxdiagnostik, nämlich Röntgenaufnahme, CT und Sonographie, wird von M. Das, Maastricht, vorgenommen. Hier wird deutlich, dass auch die bewähr- ten alten Methoden noch ihren klar definierten Platz haben und deshalb nicht zum „alten Eisen“ gelegt werden sollten. Bedingt durch technische Entwicklung hat sich bereits eine Art Quantensprung für die Röntgenaufnahme ergeben, da inzwischen direktradiographisch mit Detektorsystemen gearbeitet wird. Die erheblichen Informationsverluste über indirekte Speicherfolienbelichtung entfallen, und es kann somit auch deutlich dosisreduziert geröntgt werden. Die Thoraxröntgenuntersuchung auch und gerade als Bettaufnahme in der Intensivmedizin bzw. in der postoperativen Phase ist unverändert eine der wertvollsten radiologischen Leistungen, da sie mit geringem Aufwand erhebliche therapeutisch relevante Informationen liefert. Allein das notwendige Know-how hinsichtlich der Interpretation derartiger Aufnahmen droht (nicht nur in der Radiologie) angesichts der stetig ansteigenden Großgeräteuntersuchungszahlen zu verschwinden. Dieser fatalen Entwicklung soll dieser Beitrag entgegenwirken.

Ich hoffe, dass diese Informationen für die klinisch tätigen Kollegen eine Hilfestellung geben, auch zum Nachdenken anregen und so einen Beitrag zur weiteren Verbesserung der interdisziplinären Zusammenarbeit in der Diagnostik und Therapie thorakaler Erkrankungen leisten. Allen Autoren sei an dieser Stelle für ihre Bereitschaft mitzuwirken und die sorgfältige Darstellung der jeweiligen Thematik ausdrücklich gedankt.

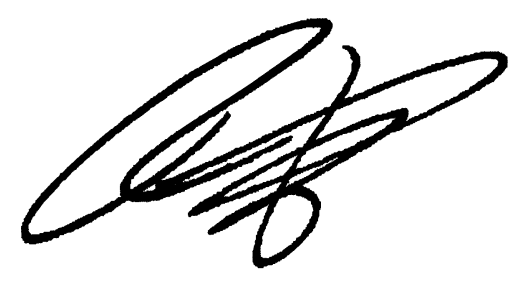

Dr. Roland C. Bittner

\section{Korrespondenzadresse Dr. R.C. Bittner}

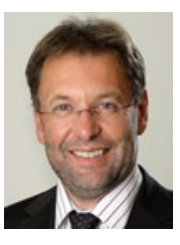

Institut für Diagnostische und Interventionelle Radiologie, Helios Klinikum Emil von Behring Walterhöferstr. 11, 14165 Berlin roland.bittner@ helios-kliniken.de

\section{COPD-Früherkennung}

Je früher, desto besser!

Die Chronisch obstruktive Lungenerkrankung (COPD) gehört zu den häufigsten chronischen Erkrankungen und hat inzwischen Platz 3 in der Todesursachenstatistik erreicht.

Eine kausale Therapie der COPD gibt es bisher nicht. Allerdings kann im Rahmen einer multimodalen Therapie aus medikamentösen und nicht medikamentösen Komponenten inzwischen eine Verzögerung des Krankheitsverlaufs erreicht werden. Diese Verbesserung der Lebenserwartung ist umso ausgeprägter je früher Therapiemaßnahmen beginnen.

Nach Daten einer Untersuchung der WHO zur Prävalenz der COPD ist etwa die Hälfte aller Erkrankungen in Deutschland nicht diagnostiziert. Dies betrifft vor allem die leichten und mittleren Krankheitsstadien, in denen der beste Langzeiteffekt der Therapie zu verzeichnen ist. Eine frühzeitigere Diagnosestellung und Therapieeinstellung muss daher medizinisch wie volkswirtschaftlich gesehen ein wichtiges gesundheitspolitisches Ziel darstellen.

Wesentlicher Grund für die verzögerte Diagnosestellung sind die unspezifischen Frühsymptome der COPD (Husten, Auswurf, Luftnot). Wichtigste Differenzialdiagnose sind Erkrankungen des HerzKreislauf-Systems. Patient wie Arzt sollte jedoch bewusst werden, dass sich hinter den genannten Leitsymptomen eine Lungenerkrankung verbergen kann, die sich mittels einer Lungenfunktionsdiagnostik diagnostizieren lässt.

Ob über die Information von Patienten und Ärzten hinaus Frühdiagnosesysteme beispielsweise in Apotheken - installiert werden können, ist unklar. Zuverlässige, einfach zu bedienende, preisgünstige Diagnosesysteme fehlen derzeit.

Quelle: Deutsche Gesellschaft für Pneumologie und Beatmungsmedizin e.V. www.pneumologie.de 